John Carroll University

Carroll Collected

2018 Faculty Bibliography

Faculty Bibliographies Community Homepage

$10-2018$

\title{
Using the Minnesota Multiphasic Personality Inventory-2-Restructured Form Cutoffs to Predict Lack of Pre-surgical Exercise
}

Brooke R. Fusco

Kent State University

Ryan J. Marek

University of Houston

Anthony M. Tarescavage

John Carroll University, atarescavage@jcu.edu

Yossef S. Ben-Porath

Kent State University

Leslie J.Heinberg

Cleveland Clinic Lerner College of Medicine

Follow this and additional works at: https://collected.jcu.edu/fac_bib_2018

Part of the Psychology Commons

\section{Recommended Citation}

Fusco, Brooke R.; Marek, Ryan J.; Tarescavage, Anthony M.; Ben-Porath, Yossef S.; and Heinberg, Leslie J., "Using the Minnesota Multiphasic Personality Inventory-2-Restructured Form Cutoffs to Predict Lack of Pre-surgical Exercise" (2018). 2018 Faculty Bibliography. 82.

https://collected.jcu.edu/fac_bib_2018/82

This Article is brought to you for free and open access by the Faculty Bibliographies Community Homepage at Carroll Collected. It has been accepted for inclusion in 2018 Faculty Bibliography by an authorized administrator of Carroll Collected. For more information, please contact connell@jcu.edu. 


\title{
Using the Minnesota Multiphasic Personality Inventory-2-Restructured Form Cutoffs to Predict Lack of Pre-surgical Exercise
}

\author{
Brooke R. Fusco · Ryan J. Marek · Anthony M. Tarescavage · Yossef S. Ben-Porath · Leslie J. Heinberg
}

\begin{abstract}
Previous studies suggest the importance of understanding what factors increase risk of lack of physical activity (PA) prior to bariatric surgery, which may increase risk of suboptimal postoperative outcomes. Therefore, the current study sought to explore which Minnesota Multiphasic Personality Inventory-2-Restructured Form (MMPI-2-RF) scales were associated with lack of pre-surgical PA. The mean age of the sample $(N=1170)$ was 45.97 years [standard deviation $(\mathrm{SD})=11.59$ ]. Bivariate correlations and relative risk ratios were utilized to examine associations between MMPI-2-RF scale scores and regular preoperative PA. Of the ten hypothesized associations, seven MMPI-2-RF scales in the internalizing and somatic domains were associated with increased risk of preoperative lack of PA. Interventions designed to increase levels of preoperative PA are especially important because individuals with higher levels of preoperative cardiorespiratory fitness experience less complications in surgery and greater weight loss postoperatively.
\end{abstract}

Keywords MMPI-2-RF $\cdot$ Bariatric surgery $\cdot$ Exercise $\cdot$ Physical activity $\cdot$ Relative risk ratios

Bariatric surgery is the most effective treatment for persons with severe obesity (i.e., for those with a BMI $>40 \mathrm{~kg} /$ $\mathrm{m}^{2}$ or for those with a BMI $\geq 35 \mathrm{~kg} / \mathrm{m}^{2}$ who have one or more serious medical comorbidities; Arterburn et al., 2013; Chang, Stoll, \& Song, 2014; Colquitt, Pickett, Loveman, \& Frampton, 2014; Gloy et al., 2013; Schauer et al., 2014; Szoka et al., 2016). Although a large percentage of bariatric patients successfully lose weight after surgery, some patients achieve suboptimal outcomes (Snyder, Nguyen, Scarbourough, Yu, \& Wilson, 2009). Thus, one goal of pre-surgical evaluations of bariatric candidates is the assessment and treatment of medical, nutritional, and psychological risk factors associated with poorer outcomes (De Luca et al.,
2016; Mechanick et al., 2013; Sogg, Lauretti, \& West-Smith, 2016).

One such risk factor involves lack of preoperative physical activity (PA). Higher levels of cardiorespiratory fitness and/or increases in PA levels prior to bariatric surgery are associated with reduced risk of surgical complications, shorter operative times, and improved healing and recovery following bariatric surgery (King \& Bond, 2013; King et al., 2008; McCullough et al., 2006; Zunker \& King, 2012). Further, research has demonstrated that patients who exercise pre- and postoperatively lose more weight after surgery than those who do not, and preoperative PA independently predicts greater postoperative PA and long-term weight loss maintenance (Bond et al., 2010; King \& Bond, 2013; King et al., 2008; Peacock, Sloan, \& Cripps, 2014). In addition to positive effects on weight loss, regular PA also contributes to regulating depression- and anxiety-related symptoms, improves body composition, and maximizes fat loss (King \& Bond, 2013; King et al., 2008). Although pre- and postsurgical exercise is associated with enhanced long-term surgical outcomes, typically, exercise habits are not addressed as a component of candidates' pre-surgical evaluations (Peacock \& Zizzi, 2011). For example, of the 123 bariatric surgery sites reviewed, Peacock and Zizzi (2011) found that only two 
required postsurgical consultations with an exercise physiologist or other exercise professional.

There are several reasons why evaluation of and counseling on exercise levels should be prioritized during bariatric surgery candidates' preoperative evaluations rather than waiting until after surgery. First, many candidates perceive several motivational, physical, and environmental barriers to starting or increasing exercise (Peacock et al., 2014; Wouters, Larsen, Zijlstra, van Ramshorst, \& Geenen, 2011), which are likely to persist after surgery if not addressed in the preoperative phase (King \& Bond, 2013; Zunker \& King, 2012). Along these lines, patients may be highly motivated prior to surgery and therefore particularly receptive to encouragement and advice on how to make positive behavior changes (King \& Bond, 2013; Zunker \& King, 2012). Additionally, initiation of or increases in PA prior to surgery reliably predict higher levels of postoperative PA (Zunker \& King, 2012). Therefore, an investigation of predictors of preoperative PA is warranted, as these predictors may be useful in guiding psychological assessments of bariatric candidates.

Pre-surgical psychological evaluations of bariatric surgery candidates have become standard practice at $90 \%$ of bariatric surgery sites and typically consist of both a clinical interview and self-report questionnaires (De Luca et al., 2016; Mechanick et al., 2013; Peacock \& Zizzi, 2011). Although specific assessment procedures vary across centers, most mental health professionals administer formal psychological testing as a component of these evaluations (Walfish, Vance, \& Fabricatore, 2007). One such standardized psychological test that has shown burgeoning use for predicting relevant outcomes among bariatric surgery candidates is the Minnesota Multiphasic Personality Inventory2-Restructured Form (MMPI-2-RF; Ben-Porath \& Tellegen, 2011). The MMPI-2-RF is a widely used psychological test made up of 338 items scored on 51 scales. Scores on these scales have been shown to be reliable and valid when used in bariatric surgery settings (Marek et al., 2013; Tarescavage et al., 2013). The MMPI-2-RF contains substantive scales that measure five fundamental domains: internalizing, externalizing, thought dysfunction, interpersonal functioning, and somatic/cognitive complaints. Of these, the internalizing and somatic/cognitive domains are of particular interest for PA research, given prior research indicating that internalizing psychopathology and somatic/cognitive complaints are associated with greater perceived barriers to PA (Peacock et al., 2014; Schuch et al., 2017; Vancampfort et al., 2016; Zabatiero et al., 2016).

Several constructs measured by scales from the MMPI2-RF internalizing domain have been linked to lack of PA and/or poor health outcomes. Demoralization measures an individual's overall dissatisfaction and unhappiness with their lives (Ben-Porath \& Tellegen, 2011), which is implicated in several medical conditions and mood disorders
(Sansone \& Sansone, 2010). Low positive emotions have been shown to be associated with lack of energy (Tellegen \& Ben-Porath, 2011), which may impede exercise motivation. Relatedly, high scores on Malaise, a scale in the somatic/ cognitive domain, are also associated with low energy. Specifically, due to excess weight, bariatric candidates often experience high levels of malaise and bodily pain, which act as barriers to exercise (Peacock et al., 2014; Zabatiero et al., 2016).

Elevations on Dysfunctional Negative Emotions characterize high levels of anxiety, which have been associated with physical inactivity (Bonnet et al., 2005). Feelings of helplessness and hopelessness (measured by Helplessness/ Hopelessness), beliefs that one is inefficacious and indecisive (measured by Inefficacy), and low self-confidence in one's abilities (measured by Self-Doubt) are also linked with lack of PA (King \& Bond, 2013; Knapen, Vancampfort, Morien, \& Marchal, 2015; Piana et al., 2013; Thomas, Hyde, Karunaratne, Kausman, \& Komesaroff, 2008). Finally, constructs measured by scales in the internalizing domain that assess more specific manifestations of feelings of anxiety, including insecurity, fear, and/or worry (i.e., Stress/Worry and Anxiety) may be associated with lack of exercise, given that obese individuals may be stigmatized for their weight and as a result, may feel too self-conscious, hesitant, or anxious to exercise in public (McIntosh, Hunter, \& Royce, 2016; Wiklund, Olsen, \& Willen, 2011).

Past research has demonstrated associations between MMPI-2-RF scales and health-related behaviors in bariatric surgery settings and primarily supports links between internalizing constructs and somatic complaints, and lack of PA (Bonnet et al., 2005; King \& Bond, 2013; Marek, BenPorath, \& Windover, 2013; McIntosh et al., 2016; Peacock et al., 2014; Schuch et al.,2017; Speck, Bond, Sarwer, \& Farrer, 2014; Wiklund et al., 2011; Zabatiero et al., 2016). Thus, associations between MMPI-2-RF scale scores and regular exercise were examined.

Based on the literature previously reviewed, it was hypothesized that higher scores on several scales in the Emotional/Internalizing Dysfunction domain would be associated with lack of preoperative PA, as well as predictive of increased risk of lack of a regular exercise program (King \& Bond, 2013; Knapen et al., 2015; McIntosh et al., 2016; Piana et al., 2013; Thomas et al., 2008; Wiklund, et al., 2011). Scales hypothesized to be associated with lack of preoperative PA and increased risk of lack of PA included: Emotional/Internalizing Dysfunction (EID), Demoralization (RCd), Low Positive Emotions (RC2), Dysfunctional Negative Emotions (RC7), Helplessness/Hopelessness (HLP), Inefficacy (NFC), Self-Doubt (SFD), Stress/Worry (STW), and Anxiety (AXY). Further, it was hypothesized that high scores on Malaise (MLS), a scale assessing low energy and fatigue, would be associated with lack of regular exercise, 
given that bariatric surgery candidates report above-average scores on this scale compared to a normative group (Marek et al., 2013). Notwithstanding these hypothesized associations, we explored the relationships between all MMPI-2-RF substantive scales and PA.

\section{Methods}

\section{Sample}

All data were collected between 2008 and 2012. The overall sample included 1268 individuals seeking bariatric surgery at the Cleveland Clinic who produced valid MMPI-2-RFs based on recommendations set forth in the test manual (VRIN-r < 80T, TRIN-r < 80T, F-r < 120T, Fp-r < 100T; Ben-Porath \& Tellegen, 2011). As part of their standard pre-surgical evaluation, patients completed a semi-structured psychiatric interview and were administered the MMPI$2-R F$. Individuals with valid $(n=1268)$ and invalid protocols $(n=43)$ did not significantly differ in terms of age, gender, or race ( $p$ 's $>.48$ ). Individuals who produced invalid protocols had significantly less years of education than individuals with valid protocols $(t=2.93, p=.003$, Cohen's $d=.39$ ). After excluding those with missing values for the regular exercise variable $(n=98)$, the final sample included 1170 individuals.

Seventy-two percent of patients were women, and $28 \%$ were men. Their mean age was 45.97 years [standard deviation $(\mathrm{SD})=11.59$ years]. The majority identified as Caucasian (65\%), with 24\% reporting as African American, 2\% as Latino, $0.2 \%$ as Asian, and $9 \%$ as other or unknown. Patients reported an average of 13.90 years of education $(\mathrm{SD}=2.52)$. At intake, the average BMI of patients was $49.17 \mathrm{~kg} / \mathrm{m}^{2}$ $(\mathrm{SD}=10.99)$. At preoperative assessment, $57 \%$ of patients had "no" regular exercise program, whereas $43 \%$ indicated having a regular exercise program.

\section{Measures}

Patients reported basic demographic information at intake into the bariatric surgery program and based on their selfreport, whether the patient reported following a regular exercise program (coded as yes/no) was entered into the database. Specifically, during the semi-structured interview, candidates were asked "Do you engage in any kind of exercise?" If answered "yes", the candidate was coded as having a regular exercise program, regardless of frequency, duration, or type of exercise.

The MMPI-2-RF (Ben-Porath \& Tellegen, 2011; Tellegen \& Ben-Porath, 2011) is a widely used psychological test made up of 338 items scored on 51 scales. The MMPI2-RF contains nine validity scales, which measure random and fixed all-true or all-false responding, as well as over- or under-reporting. The test also contains 42 substantive scales that assess personality and psychopathology. MMPI-2-RF scores have been shown to be reliable and valid when used in bariatric surgery settings (Marek et al., 2013; Tarescavage et al., 2013) and comparison group data for bariatric surgery patients are available (Tellegen \& Ben-Porath, 2011).

\section{Procedures}

MMPI-2-RF scales scores, patient demographics, and exercise behavior information were collected during the standard pre-surgical evaluation by a doctoral level clinical psychologist. This information was coded via a retrospective chart review by trained research assistants. To maintain consistency and reduce errors, all coded data were double entered. The research assistants achieved an average inter-rater reliability statistic of .96 (range .81-1.00). The use of patient information for research purposes was approved by both the Cleveland Clinic and Kent State University's Institutional Review Boards.

\section{Results}

\section{Descriptive Findings and Correlations}

To examine our hypotheses, point-biserial bivariate correlations between preoperative regular exercise and MMPI-2-RF scales were first examined. Only correlations above .15 were considered meaningful for interpretation. Although .20 is the traditional criterion for clinical samples (Anastasi \& Urbina, 1997), bariatric surgery candidates are more likely to minimize psychological distress during pre-surgical evaluation to be recommended for surgery (Ambwani et al., 2013). This minimization results in range restriction and lower scale scores, as well as attenuated correlations with external criteria, justifying the use of lower thresholds to interpret correlations (Marek et al., 2015).

Means and SD for all MMPI-2-RF scale scores in the study, as well as correlations between regular exercise and MMPI-2-RF scales, are presented for the full sample $(N=1170)$ in Table 1 . As hypothesized, higher scores on RC2 and MLS were associated with lack of a regular exercise program. EID, RCd, RC7, NFC, SFD, STW, and AXY were not meaningfully associated with regular exercise as hypothesized.

\section{Logistic Regression}

A logistic regression analysis was next performed to examine the joint prediction of exercise behavior by RC2 and MLS. These MMPI-2-RF scales were chosen because they 
Table 1 MMPI-2-RF scales mean and standard deviations, associations between pre-surgical MMPI-2-RF scores and regular exercise, and relative risk ratios for non-exercisers $(N=1170)$

\begin{tabular}{|c|c|c|c|c|c|c|c|c|c|}
\hline \multirow[t]{2}{*}{ Scale name } & \multicolumn{2}{|c|}{$\begin{array}{l}\text { Descrip- } \\
\text { tives }\end{array}$} & \multirow{2}{*}{$\begin{array}{l}\text { Correlations } \\
r\end{array}$} & \multicolumn{6}{|c|}{ Relative risk ratios } \\
\hline & M & SD & & Cutoff score & $\operatorname{SR}(n)$ & Risk if elevated & $\begin{array}{l}\text { Risk } \\
\text { if not } \\
\text { elevated }\end{array}$ & RRR & $95 \% \mathrm{CI}$ \\
\hline Emotional/Internalizing Dysfunction & 50.1 & 10.9 & $-0.10 * *$ & 65 & $10.7 \%(125)$ & $63.2 \%$ & $56.7 \%$ & 1.116 & $(0.97,1.29)$ \\
\hline Emotional/Internalizing Dysfunction & & & & 60 & $16.9 \%(198)$ & $65.2 \%$ & $55.8 \%$ & 1.168 & $(1.04,1.31)$ \\
\hline Emotional/Internalizing Dysfunction & & & & 55 & $28.7 \%(336)$ & $61.9 \%$ & $55.5 \%$ & 1.115 & $(1.01,1.24)$ \\
\hline Thought Dysfunction & 47.6 & 8.9 & -0.02 & 65 & $4.7 \%(55)$ & $50.9 \%$ & $57.7 \%$ & 0.883 & $(0.68,1.15)$ \\
\hline Thought Dysfunction & & & & 60 & $11.0 \%(129)$ & $60.5 \%$ & $57.0 \%$ & 1.061 & $(0.91,1.23)$ \\
\hline Thought Dysfunction & & & & 55 & $18.0 \%(211)$ & $64.0 \%$ & $55.9 \%$ & 1.145 & $(1.02,1.29)$ \\
\hline Behavioral/Externalizing Dysfunction & 45.3 & 8.5 & 0.01 & 65 & $2.8 \%(33)$ & $48.5 \%$ & $57.6 \%$ & 0.842 & $(0.59,1.20)$ \\
\hline Behavioral/Externalizing Dysfunction & & & & 60 & $5.9 \%(69)$ & $58.0 \%$ & $57.3 \%$ & 1.012 & $(0.82,1.24)$ \\
\hline Behavioral/Externalizing Dysfunction & & & & 55 & $15.2 \%(178)$ & $58.4 \%$ & $57.2 \%$ & 1.022 & $(0.89,1.17)$ \\
\hline Demoralization & 51.3 & 9.9 & $-0.10 * *$ & 65 & $11.3 \%(132)$ & $63.6 \%$ & $56.6 \%$ & 1.125 & $(0.98,1.29)$ \\
\hline Demoralization & & & & 60 & $18.5 \%(216)$ & $66.2 \%$ & $55.3 \%$ & 1.196 & $(1.07,1.34)$ \\
\hline Demoralization & & & & 55 & $32.1 \%(276)$ & $\mathrm{n} / \mathrm{c}$ & $\mathrm{n} / \mathrm{c}$ & $\mathrm{n} / \mathrm{c}$ & $\mathrm{n} / \mathrm{c}$ \\
\hline Somatic Complaints & 57.2 & 11.6 & $-0.07 *$ & 65 & $24.8 \%(290)$ & $61.4 \%$ & $56.0 \%$ & 1.096 & $(0.98,1.22)$ \\
\hline Somatic Complaints & & & & 60 & $38.3 \%(448)$ & $\mathrm{n} / \mathrm{c}$ & $\mathrm{n} / \mathrm{c}$ & $\mathrm{n} / \mathrm{c}$ & $\mathrm{n} / \mathrm{c}$ \\
\hline Somatic Complaints & & & & 55 & $55.0 \%(644)$ & $\mathrm{n} / \mathrm{c}$ & $\mathrm{n} / \mathrm{c}$ & $\mathrm{n} / \mathrm{c}$ & $\mathrm{n} / \mathrm{c}$ \\
\hline Low Positive Emotions & 51.9 & 11.1 & $-0.15^{* *}$ & 65 & $15.4 \%(180)$ & $66.7 \%$ & $55.7 \%$ & 1.198 & $(1.07,1.35)$ \\
\hline Low Positive Emotions & & & & 60 & $23.0 \%(269)$ & $66.5 \%$ & $54.6 \%$ & 1.219 & $(1.10,1.35)$ \\
\hline Low Positive Emotions & & & & 55 & $30.8 \%(360)$ & $\mathrm{n} / \mathrm{c}$ & $\mathrm{n} / \mathrm{c}$ & $\mathrm{n} / \mathrm{c}$ & $\mathrm{n} / \mathrm{c}$ \\
\hline Cynicism & 47.9 & 10.3 & $-0.07 *$ & 65 & $9.8 \%(115)$ & $67.0 \%$ & $56.3 \%$ & 1.189 & $(1.03,1.37)$ \\
\hline Cynicism & & & & 60 & $14.2 \%(166)$ & $66.3 \%$ & $55.9 \%$ & 1.186 & $(1.05,1.34)$ \\
\hline Cynicism & & & & 55 & $19.8 \%(232)$ & $63.8 \%$ & $55.8 \%$ & 1.144 & $(1.02,1.28)$ \\
\hline Antisocial Behavior & 46.4 & 8.5 & -0.01 & 65 & $3.9 \%(46)$ & $54.3 \%$ & $57.5 \%$ & 0.946 & $(0.72,1.24)$ \\
\hline Antisocial Behavior & & & & 60 & $5.5 \%(64)$ & $56.3 \%$ & $57.4 \%$ & 0.980 & $(0.78,1.22)$ \\
\hline Antisocial Behavior & & & & 55 & $14.3 \%(167)$ & $57.5 \%$ & $57.3 \%$ & 1.003 & $(0.87,1.15)$ \\
\hline Ideas of Persecution & 50.7 & 9.9 & -0.01 & 65 & $10.9 \%(128)$ & $58.6 \%$ & $57.2 \%$ & 1.024 & $(0.88,1.20)$ \\
\hline Ideas of Persecution & & & & 60 & $22.1 \%(258)$ & $57.8 \%$ & $57.2 \%$ & 1.009 & $(0.90,1.14)$ \\
\hline Ideas of Persecution & & & & 55 & $42.6 \%(499)$ & $\mathrm{n} / \mathrm{c}$ & $\mathrm{n} / \mathrm{c}$ & $\mathrm{n} / \mathrm{c}$ & $\mathrm{n} / \mathrm{c}$ \\
\hline Dysfunctional Negative Emotions & 46.4 & 9.4 & -0.04 & 65 & $5.5 \%(64)$ & $62.5 \%$ & $57.1 \%$ & 1.095 & $(0.90,1.33)$ \\
\hline Dysfunctional Negative Emotions & & & & 60 & $9.8 \%(115)$ & $57.4 \%$ & $57.3 \%$ & 1.001 & $(0.85,1.18)$ \\
\hline Dysfunctional Negative Emotions & & & & 55 & $17.2 \%(201)$ & $57.7 \%$ & $57.3 \%$ & 1.008 & $(0.88,1.15)$ \\
\hline Aberrant Experiences & 47.1 & 8.4 & -0.02 & 65 & $4.4 \%(51)$ & $52.9 \%$ & $57.6 \%$ & 0.920 & $(0.71,1.20)$ \\
\hline Aberrant Experiences & & & & 60 & $7.4 \%(87)$ & $59.8 \%$ & $57.2 \%$ & 1.046 & $(0.87,1.25)$ \\
\hline Aberrant Experiences & & & & 55 & $18.5 \%(216)$ & $60.6 \%$ & $56.6 \%$ & 1.071 & $(0.95,1.21)$ \\
\hline Hypomanic Activation & 43.3 & 8.6 & 0.02 & 65 & $2.1 \%(25)$ & $64.0 \%$ & $57.2 \%$ & 1.119 & $(0.83,1.51)$ \\
\hline Hypomanic Activation & & & & 60 & $3.8 \%(44)$ & $63.6 \%$ & $57.1 \%$ & 1.114 & $(0.89,1.40)$ \\
\hline Hypomanic Activation & & & & 55 & $9.6 \%(112)$ & $64.3 \%$ & $56.6 \%$ & 1.135 & $(0.98,1.32)$ \\
\hline Malaise & 64.4 & 12.2 & $-0.18 * *$ & 65 & $44.5 \%(521)$ & $\mathrm{n} / \mathrm{c}$ & $\mathrm{n} / \mathrm{c}$ & $\mathrm{n} / \mathrm{c}$ & $\mathrm{n} / \mathrm{c}$ \\
\hline Malaise & & & & 60 & $60.9 \%(713)$ & $\mathrm{n} / \mathrm{c}$ & $\mathrm{n} / \mathrm{c}$ & $\mathrm{n} / \mathrm{c}$ & $\mathrm{n} / \mathrm{c}$ \\
\hline Malaise & & & & 55 & $77.4 \%(906)$ & $\mathrm{n} / \mathrm{c}$ & $\mathrm{n} / \mathrm{c}$ & $\mathrm{n} / \mathrm{c}$ & $\mathrm{n} / \mathrm{c}$ \\
\hline Gastrointestinal Complaints & 52.5 & 11.6 & -0.04 & 65 & $12.6 \%(148)$ & $60.8 \%$ & $56.8 \%$ & 1.070 & $(0.93,1.23)$ \\
\hline Gastrointestinal Complaints & & & & 60 & $26.8 \%(313)$ & $59.1 \%$ & $56.7 \%$ & 1.042 & $(0.93,1.16)$ \\
\hline Gastrointestinal Complaints & & & & 55 & $26.8 \%(313)$ & $59.1 \%$ & $56.7 \%$ & 1.042 & $(0.93,1.16)$ \\
\hline Head Pain Complaints & 55.8 & 10.4 & -0.05 & 65 & $24.4 \%(286)$ & $60.8 \%$ & $56.2 \%$ & 1.082 & $(0.97,1.21)$ \\
\hline Head Pain Complaints & & & & 60 & $24.4 \%(286)$ & $60.8 \%$ & $56.2 \%$ & 1.082 & $(0.97,1.21)$ \\
\hline Head Pain Complaints & & & & 55 & $47.4 \%(555)$ & $\mathrm{n} / \mathrm{c}$ & $\mathrm{n} / \mathrm{c}$ & $\mathrm{n} / \mathrm{c}$ & $\mathrm{n} / \mathrm{c}$ \\
\hline
\end{tabular}


Table 1 (continued)

\begin{tabular}{|c|c|c|c|c|c|c|c|c|c|}
\hline \multirow[t]{2}{*}{ Scale name } & \multicolumn{2}{|c|}{$\begin{array}{l}\text { Descrip- } \\
\text { tives }\end{array}$} & \multirow{2}{*}{$\begin{array}{l}\text { Correlations } \\
r\end{array}$} & \multicolumn{6}{|c|}{ Relative risk ratios } \\
\hline & M & SD & & Cutoff score & $\operatorname{SR}(n)$ & Risk if elevated & $\begin{array}{l}\text { Risk } \\
\text { if not } \\
\text { elevated }\end{array}$ & RRR & $95 \% \mathrm{CI}$ \\
\hline Cognitive Complaints & 50.7 & 11.0 & $-0.06^{*}$ & 65 & $10.3 \%(121)$ & $58.7 \%$ & $57.2 \%$ & 1.026 & $(0.88,1.20)$ \\
\hline Cognitive Complaints & & & & 60 & $16.8 \%(196)$ & $63.8 \%$ & $56.1 \%$ & 1.138 & $(1.01,1.28)$ \\
\hline Cognitive Complaints & & & & 55 & $24.2 \%(283)$ & $64.7 \%$ & $55.0 \%$ & 1.175 & $(1.06,1.31)$ \\
\hline Suicidal/Death Ideation & 48.2 & 8.5 & $-0.07 *$ & 65 & $13.3 \%(156)$ & $66.7 \%$ & $55.9 \%$ & 1.192 & $(1.05,1.35)$ \\
\hline Suicidal/Death Ideation & & & & 60 & $13.3 \%(156)$ & $66.7 \%$ & $55.9 \%$ & 1.192 & $(1.05,1.35)$ \\
\hline Suicidal/Death Ideation & & & & 55 & $13.3 \%(156)$ & $66.7 \%$ & $55.9 \%$ & 1.192 & $(1.05,1.35)$ \\
\hline Helplessness/Hopelessness & 47.0 & 9.4 & $-0.10 * *$ & 65 & $4.9 \%(57)$ & $77.2 \%$ & $56.3 \%$ & 1.370 & $(1.18,1.59)$ \\
\hline Helplessness/Hopelessness & & & & 60 & $14.4 \%(169)$ & $65.7 \%$ & $55.9 \%$ & 1.174 & $(1.04,1.33)$ \\
\hline Helplessness/Hopelessness & & & & 55 & $14.4 \%(169)$ & $65.7 \%$ & $55.9 \%$ & 1.174 & $(1.04,1.33)$ \\
\hline Self-Doubt & 51.6 & 10.8 & $-0.06^{*}$ & 65 & $17.9 \%(209)$ & $62.7 \%$ & $56.2 \%$ & 1.115 & $(0.99,1.26)$ \\
\hline Self-Doubt & & & & 60 & $17.9 \%(209)$ & $62.7 \%$ & $56.2 \%$ & 1.115 & $(0.99,1.26)$ \\
\hline Self-Doubt & & & & 55 & $33.2 \%(388)$ & $\mathrm{n} / \mathrm{c}$ & $\mathrm{n} / \mathrm{c}$ & $\mathrm{n} / \mathrm{c}$ & $\mathrm{n} / \mathrm{c}$ \\
\hline Inefficacy & 48.3 & 10.1 & $-0.07 *$ & 65 & $7.0 \%(82)$ & $67.1 \%$ & $56.6 \%$ & 1.185 & $(1.01,1.39)$ \\
\hline Inefficacy & & & & 60 & $11.3 \%(132)$ & $68.2 \%$ & $56.0 \%$ & 1.218 & $(1.07,1.39)$ \\
\hline Inefficacy & & & & 55 & $17.4 \%(204)$ & $65.2 \%$ & $55.7 \%$ & 1.171 & $(1.04,1.31)$ \\
\hline Stress/Worry & 50.2 & 10.0 & $-0.07 *$ & 65 & $13.8 \%(162)$ & $65.4 \%$ & $56.1 \%$ & 1.167 & $(1.03,1.32)$ \\
\hline Stress/Worry & & & & 60 & $13.8 \%(162)$ & $65.4 \%$ & $56.1 \%$ & 1.167 & $(1.03,1.32)$ \\
\hline Stress/Worry & & & & 55 & $28.5 \%(334)$ & $63.8 \%$ & $54.8 \%$ & 1.164 & $(1.05,1.29)$ \\
\hline Anxiety & 49.1 & 10.1 & -0.05 & 65 & $7.9 \%(92)$ & $58.7 \%$ & $57.2 \%$ & 1.026 & $(0.86,1.23)$ \\
\hline Anxiety & & & & 60 & $7.9 \%(92)$ & $58.7 \%$ & $57.2 \%$ & 1.026 & $(0.86,1.23)$ \\
\hline Anxiety & & & & 55 & $24.6 \%(288)$ & $64.6 \%$ & $55.0 \%$ & 1.174 & $(1.06,1.30)$ \\
\hline Anger Proneness & 47.8 & 9.6 & -0.05 & 65 & $9.3 \%(109)$ & $62.4 \%$ & $56.8 \%$ & 1.098 & $(0.94,1.28)$ \\
\hline Anger Proneness & & & & 60 & $9.3 \%(109)$ & $62.4 \%$ & $56.8 \%$ & 1.098 & $(0.94,1.28)$ \\
\hline Anger Proneness & & & & 55 & $14.6 \%(171)$ & $60.2 \%$ & $56.9 \%$ & 1.059 & $(0.93,1.21)$ \\
\hline Behavior-Restricting Fears & 48.6 & 8.8 & -0.05 & 65 & $3.9 \%(46)$ & $65.2 \%$ & $57.0 \%$ & 1.144 & $(0.92,1.42)$ \\
\hline Behavior-Restricting Fears & & & & 60 & $11.3 \%(132)$ & $66.7 \%$ & $56.2 \%$ & 1.187 & $(1.04,1.35)$ \\
\hline Behavior-Restricting Fears & & & & 55 & $33.6 \%(393)$ & $\mathrm{n} / \mathrm{c}$ & $\mathrm{n} / \mathrm{c}$ & $\mathrm{n} / \mathrm{c}$ & $\mathrm{n} / \mathrm{c}$ \\
\hline Multiple Specific Fears & 49.7 & 8.6 & -0.05 & 65 & $9.5 \%(111)$ & $54.1 \%$ & $57.7 \%$ & 0.937 & $(0.78,1.12)$ \\
\hline Multiple Specific Fears & & & & 60 & $9.5 \%(111)$ & $54.1 \%$ & $57.7 \%$ & 0.937 & $(0.78,1.12)$ \\
\hline Multiple Specific Fears & & & & 55 & $17.4 \%(204)$ & $60.3 \%$ & $56.7 \%$ & 1.063 & $(0.94,1.20)$ \\
\hline Juvenile Conduct Problems & 48.7 & 10.0 & 0.00 & 65 & $5.6 \%(65)$ & $55.4 \%$ & $57.5 \%$ & 0.964 & $(0.77,1.21)$ \\
\hline Juvenile Conduct Problems & & & & 60 & $14.1 \%(165)$ & $57.0 \%$ & $57.4 \%$ & 0.992 & $(0.86,1.14)$ \\
\hline Juvenile Conduct Problems & & & & 55 & $26.9 \%(315)$ & $57.5 \%$ & $57.3 \%$ & 1.003 & $(0.90,1.12)$ \\
\hline Substance Abuse & & & & 65 & $0.5 \%(6)$ & $\mathrm{n} / \mathrm{c}$ & $\mathrm{n} / \mathrm{c}$ & $\mathrm{n} / \mathrm{c}$ & $\mathrm{n} / \mathrm{c}$ \\
\hline Substance Abuse & 44.4 & 5.5 & 0.00 & 60 & $2.2 \%(26)$ & $57.7 \%$ & $57.3 \%$ & 1.006 & $(0.72,1.40)$ \\
\hline Substance Abuse & & & & 55 & $9.7 \%(114)$ & $56.1 \%$ & $57.5 \%$ & 0.977 & $(0.82,1.16)$ \\
\hline Aggression & 44.7 & 8.3 & -0.03 & 65 & $2.3 \%(27)$ & $63.0 \%$ & $57.2 \%$ & 1.100 & $(0.82,1.48)$ \\
\hline Aggression & & & & 60 & $6.3 \%(74)$ & $59.5 \%$ & $57.2 \%$ & 1.039 & $(0.86,1.26)$ \\
\hline Aggression & & & & 55 & $13.7 \%(160)$ & $60.0 \%$ & $56.9 \%$ & 1.054 & $(0.92,1.21)$ \\
\hline Activation & 44.5 & 9.3 & $0.06^{*}$ & 65 & $4.4 \%(52)$ & $53.8 \%$ & $57.5 \%$ & 0.936 & $(0.72,1.21)$ \\
\hline Activation & & & & 60 & $4.4 \%(52)$ & $53.8 \%$ & $57.5 \%$ & 0.936 & $(0.72,1.21)$ \\
\hline Activation & & & & 55 & $10.3 \%(121)$ & $57.9 \%$ & $57.3 \%$ & 1.010 & $(0.86,1.19)$ \\
\hline Family Problems & 47.4 & 9.5 & -0.02 & 65 & $6.2 \%(72)$ & $61.1 \%$ & $57.1 \%$ & 1.070 & $(0.88,1.30)$ \\
\hline Family Problems & & & & 60 & $10.3 \%(120)$ & $57.5 \%$ & $57.3 \%$ & 1.003 & $(0.85,1.18)$ \\
\hline Family Problems & & & & 55 & $16.4 \%(192)$ & $55.2 \%$ & $57.8 \%$ & 0.956 & $(0.83,1.10)$ \\
\hline Interpersonal Passivity & 48.5 & 9.5 & -0.03 & 65 & $7.4 \%(86)$ & $64.0 \%$ & $56.8 \%$ & 1.125 & $(0.95,1.33)$ \\
\hline
\end{tabular}


Table 1 (continued)

\begin{tabular}{|c|c|c|c|c|c|c|c|c|c|}
\hline \multirow[t]{2}{*}{ Scale name } & \multicolumn{2}{|c|}{$\begin{array}{l}\text { Descrip- } \\
\text { tives }\end{array}$} & \multirow{2}{*}{$\begin{array}{l}\text { Correlations } \\
r\end{array}$} & \multicolumn{6}{|c|}{ Relative risk ratios } \\
\hline & M & SD & & Cutoff score & $\operatorname{SR}(n)$ & Risk if elevated & $\begin{array}{l}\text { Risk } \\
\text { if not } \\
\text { elevated }\end{array}$ & RRR & $95 \% \mathrm{CI}$ \\
\hline Interpersonal Passivity & & & & 60 & $12.9 \%(151)$ & $60.3 \%$ & $56.9 \%$ & 1.059 & $(0.92,1.22)$ \\
\hline Interpersonal Passivity & & & & 55 & $20.7 \%(242)$ & $59.5 \%$ & $56.8^{\wedge}$ & 1.048 & $(0.93,1.18)$ \\
\hline Social Avoidance & 51.5 & 10.5 & $-0.11 * *$ & 65 & $17.3 \%(202)$ & $65.8 \%$ & $55.6 \%$ & 1.185 & $(1.06,1.33)$ \\
\hline Social Avoidance & & & & 60 & $17.3 \%(202)$ & $65.8 \%$ & $55.6 \%$ & 1.185 & $(1.06,1.33)$ \\
\hline Social Avoidance & & & & 55 & $32.3 \%(378)$ & $63.0 \%$ & $54.7 \%$ & 1.152 & $(1.04,1.27)$ \\
\hline Shyness & 46.9 & 9.1 & -0.06 & 65 & $8.5 \%(100)$ & $59.0 \%$ & $57.2 \%$ & 1.032 & $(0.87,1.22)$ \\
\hline Shyness & & & & 60 & $8.5 \%(100)$ & $59.0 \%$ & $57.2 \%$ & 1.032 & $(0.87,1.22)$ \\
\hline Shyness & & & & 55 & $14.9 \%(174)$ & $57.5 \%$ & $57.3 \%$ & 1.002 & $(0.87,1.15)$ \\
\hline Disaffiliativeness & 49.5 & 9.8 & -0.04 & 65 & $10.5 \%(123)$ & $61.0 \%$ & $56.9 \%$ & 1.071 & $(0.92,1.25)$ \\
\hline Disaffiliativeness & & & & 60 & $10.5 \%(123)$ & $61.0 \%$ & $56.9 \%$ & 1.071 & $(0.92,1.25)$ \\
\hline Disaffiliativeness & & & & 55 & $28.4 \%(332)$ & $60.5 \%$ & $56.1 \%$ & 1.079 & $(0.97,1.20)$ \\
\hline Aggressiveness & 49.9 & 9.0 & -0.00 & 65 & $9.4 \%(110)$ & $63.6 \%$ & $56.7 \%$ & 1.122 & $(0.97,1.30)$ \\
\hline Aggressiveness & & & & 60 & $17.1 \%(200)$ & $61.0 \%$ & $56.6 \%$ & 1.078 & $(0.95,1.22)$ \\
\hline Aggressiveness & & & & 55 & $27.1 \%(317)$ & $59.6 \%$ & $56.5 \%$ & 1.055 & $(0.95,1.18)$ \\
\hline Psychoticism & 46.9 & 8.9 & -0.04 & 65 & $4.2 \%(49)$ & $55.1 \%$ & $57.4 \%$ & 0.959 & $(0.74,1.24)$ \\
\hline Psychoticism & & & & 60 & $6.7 \%(78)$ & $60.3 \%$ & $57.1 \%$ & 1.054 & $(0.87,1.27)$ \\
\hline Psychoticism & & & & 55 & $19.7 \%(230)$ & $62.2 \%$ & $56.2 \%$ & 1.107 & $(0.99,1.24)$ \\
\hline Disconstraint & 44.6 & 8.1 & 0.03 & 65 & $2.1 \%(25)$ & $48.0 \%$ & $57.6 \%$ & 0.834 & $(0.55,1.26)$ \\
\hline Disconstraint & & & & 60 & $3.6 \%(42)$ & $50.0 \%$ & $57.6 \%$ & 0.868 & $(0.64,1.18)$ \\
\hline Disconstraint & & & & 55 & $10.1 \%(118)$ & $55.9 \%$ & $57.5 \%$ & 0.973 & $(0.82,1.15)$ \\
\hline Negative Emotionality/Neuroticism & 48.7 & 9.9 & $-0.07 *$ & 65 & $8.1 \%(95)$ & $64.2 \%$ & $56.7 \%$ & 1.132 & $(0.97,1.33)$ \\
\hline Negative Emotionality/Neuroticism & & & & 60 & $11.5 \%(135)$ & $63.7 \%$ & $56.5 \%$ & 1.127 & $(0.98,1.29)$ \\
\hline Negative Emotionality/Neuroticism & & & & 55 & $23.1 \%(270)$ & $62.2 \%$ & $55.9 \%$ & 1.113 & $(1.00,1.24)$ \\
\hline Introversion/Low Positive Emotionality & 53.1 & 10.8 & $-0.14 * *$ & 65 & $14.5 \%(170)$ & $68.8 \%$ & $55.4 \%$ & 1.242 & $(1.11,1.39)$ \\
\hline Introversion/Low Positive Emotionality & & & & 60 & $25.4 \%(297)$ & $64.0 \%$ & $55.1 \%$ & 1.161 & $(1.05,1.29)$ \\
\hline Introversion/Low Positive Emotionality & & & & 55 & $33.9 \%(397)$ & $\mathrm{n} / \mathrm{c}$ & $\mathrm{n} / \mathrm{c}$ & $\mathrm{n} / \mathrm{c}$ & $\mathrm{n} / \mathrm{c}$ \\
\hline
\end{tabular}

Scales in bold are those hypothesized to be associated with lack of preoperative PA and increased risk of lack of PA. n/c $=$ not calculated; relative risk ratios were only calculated for scales and cutoffs with selection ratios ranging from 2 to $30 \%$.

$S R$ selection ratio, $R R R$ relative risk ratio, $C I$ confidence interval

*Significant at the .05 level

**Significant at the .01 level

emerged as significant in the correlational analyses. We therefore wanted to determine their independent contribution to the prediction of likelihood of engaging in regular exercise. The overall model was statistically significant $\left(\chi^{2}=42.09, p<.001\right)$. However, only MLS was a significant, unique predictor $(p<.001)$, whereas $\mathrm{RC} 2$ was marginally significant $(p=.085)$.

\section{Relative Risk Ratios}

Lastly, we utilized relative risk ratios (RRRs) to quantify the risk of not engaging in a regular pre-surgical exercise program associated with various MMPI-2-RF scores. MMPI2-RF T-scores were dichotomized using the traditional cutoff of $65 \mathrm{~T}$, as well as cutoffs of 60T and 55T. We examined these lower cutoffs because of the restricted range of scores in this setting discussed earlier. Further, past research has supported the use of lower MMPI-2-RF scale score cutoffs for enhanced predictive validity in other studies of bariatric surgery candidates (Tarescavage et al., 2013; Tarescavage, Wygant, Boutacoff, \& Ben-Porath, 2013).

RRRs for hypothesized MMPI-2-RF scales are also presented in Table 1. To orient the reader to the analyses, the selection ratio (SR) represents the percentage of individuals who produced clinically elevated scores above the designated cutoff level. Only scale score cutoffs with SRs ranging from 2 to $30 \%$ were calculated and included to reduce the likelihood of obtaining results affected by outliers or false 
positive results, respectively. After each SR, in parentheses, are frequencies of those falling at or above the designated cutoff. For example, the findings for EID (row 2 of Table 1) indicate that $16.9 \%$ of the sample $(n=198)$ scored at or above $60 \mathrm{~T}$ on this scale. The risk of not exercising if EID $\geq 60$ is $65.2 \%$ and the risk if EID $<60$ is $55.8 \%$. Dividing the risk whether elevated by the risk not elevated yields an RRR of 1.168, indicating that a score of 60 or higher on EID increases risk of lack of regular exercise by $16.8 \%$ (Andrade, 2015; Streiner, 1998). Because the RRR's 95\% confidence interval (CI 1.04, 1.31) does not overlap with the value of 1.0, the finding is statistically significant (Andrade, 2015; Bewick, Cheek, \& Ball, 2004). A CI that includes 1.0 in its range indicates the possibility that there is an equal risk of not exercising between those producing elevations and those who do not, and therefore, we would fail to reject the null hypothesis that the risk is equal across the two groups (Andrade, 2015; Bewick et al., 2004). It is important to note that the base rate of no exercise preoperatively in the current sample is approximately $50 \%$, meaning the maximum possible value of the RRR is approximately 2 . That is, even if all individuals who elevated a scale did not exercise, the maximum RRR we would achieve would be about 2 .

Of the ten hypothesized associations, seven were significant. In support of our hypotheses, these RRR indicates that elevations on EID, RCd, RC2, HLP, NFC, STW, and AXY are associated with increased risk of lack of regular exercise preoperatively. For example, elevations on RC2 increase risk of lack of pre-surgical exercise by almost $22 \%$ and elevations on HLP increase this risk by $37 \%$. Contrary to our hypotheses, associations between RC7 and SFD with increased risk of lack of exercise were not supported. Further, although elevations on MLS were significantly associated with increased risk of lack of regular exercise, the SR was outside the $2-30 \%$ range and these results were, therefore, not interpreted.

Table 1 also includes results of our exploratory analyses on the other MMPI-2-RF substantive scales. In these exploratory analyses, we found statistically significant RRRs for elevations on the following scales: Thought Dysfunction (1.145 if THD $\geq 55$ ), Cynicism (1.189 if RC3 $\geq 65$ ), Cognitive Complaints (1.138 if COG $\geq 60$ ), Suicidal/Death Ideation (1.192 if SUI $\geq 55$ ), Social Avoidance (1.185 if SUI $\geq 60$ ) and Introversion/Neuroticism-Revised (1.242 if INTR-r $\geq 65$ ).

Post hoc RRR analyses were conducted to explore the utility of scale elevation combinations of RC2 and MLS using cutoffs of $60 \mathrm{~T}$ and $65 \mathrm{~T}$ in the prediction of regular exercise. No RRR combinations could be calculated for cutoffs of 55T because this yielded out-of-range selection ratios. All calculated combinations of scale elevations yielded significant RRRs, ranging from 37 to $45 \%$ increased risk of lack of exercise (see Table 2). These estimates of risk were higher than when RC2 was utilized individually,
Table 2 Relative risk ratios for combinations of low positive emotions and Malaise elevations

\begin{tabular}{|c|c|c|c|c|c|c|}
\hline Scale combinations & Cutoff score & $\operatorname{SR}(n)$ & $\begin{array}{l}\text { Risk if both } \\
\text { scales elevated } \\
(n)\end{array}$ & $\begin{array}{l}\text { Risk if neither } \\
\text { scale elevated } \\
(n)\end{array}$ & RRR & $95 \% \mathrm{CI}$ \\
\hline Low Positive Emotions & 65 & & & & & \\
\hline Malaise & 65 & $19.6 \%(151)$ & $66.9 \%(101)$ & $48.9 \%(303)$ & 1.369 & $(1.19,1.57)$ \\
\hline Low Positive Emotions & 60 & & & & & \\
\hline Malaise & 60 & $36.1 \%(244)$ & $\mathrm{n} / \mathrm{c}$ & $\mathrm{n} / \mathrm{c}$ & $\mathrm{n} / \mathrm{c}$ & $\mathrm{n} / \mathrm{c}$ \\
\hline Low Positive Emotions & 55 & & & & & \\
\hline Malaise & 55 & $58.3 \%(336)$ & $\mathrm{n} / \mathrm{c}$ & $\mathrm{n} / \mathrm{c}$ & $\mathrm{n} / \mathrm{c}$ & $\mathrm{n} / \mathrm{c}$ \\
\hline Low Positive Emotions & 65 & & & & & \\
\hline Malaise & 60 & $27.5 \%(169)$ & $67.5 \%(114)$ & $46.6 \%(208)$ & 1.446 & $(1.25,1.67)$ \\
\hline Low Positive Emotions & 65 & & & & & \\
\hline Malaise & 55 & $40.5 \%(178)$ & $\mathrm{n} / \mathrm{c}$ & $\mathrm{n} / \mathrm{c}$ & $\mathrm{n} / \mathrm{c}$ & $\mathrm{n} / \mathrm{c}$ \\
\hline Low Positive Emotions & 60 & & & & & \\
\hline Malaise & 65 & $26.5 \%(215)$ & $67.9 \%(146)$ & $48.6 \%(289)$ & 1.398 & $(1.24,1.58)$ \\
\hline Low Positive Emotions & 55 & & & & & \\
\hline Malaise & 65 & $32.6 \%(270)$ & $\mathrm{n} / \mathrm{c}$ & $\mathrm{n} / \mathrm{c}$ & $\mathrm{n} / \mathrm{c}$ & $\mathrm{n} / \mathrm{c}$ \\
\hline Low Positive Emotions & 60 & & & & & \\
\hline Malaise & 55 & $50.5 \%(260)$ & $\mathrm{n} / \mathrm{c}$ & $\mathrm{n} / \mathrm{c}$ & $\mathrm{n} / \mathrm{c}$ & $\mathrm{n} / \mathrm{c}$ \\
\hline Low Positive Emotions & 55 & & & & & \\
\hline Malaise & 60 & $43.3 \%(311)$ & $\mathrm{n} / \mathrm{c}$ & $\mathrm{n} / \mathrm{c}$ & $\mathrm{n} / \mathrm{c}$ & $\mathrm{n} / \mathrm{c}$ \\
\hline
\end{tabular}

$\mathrm{n} / \mathrm{c}=$ not calculated; relative risk ratios were only calculated for scales and cutoffs with selection ratios ranging from 2 to $30 \%$

$S R$ selection ratio, $R R R$ relative risk ratio, $C I$ confidence interval 
which ranged from 20 to $22 \%$ increased risk. Moreover, these analyses allowed the usage of MLS, for which RRRs could not be produced in original analyses. For example, an individual who produces a clinical elevation (65T or higher) on RC2 and an elevation of 60T or higher on MLS yields the highest estimate of risk, increasing the likelihood of lack of exercise by nearly $45 \%$, while an elevation of $60 \mathrm{~T}$ or higher on RC2 and $65 \mathrm{~T}$ or higher on MLS increases risk of lack of exercise by $40 \%$.

\section{Discussion}

The purpose of the present study was to investigate associations between MMPI-2-RF scores and regular exercise among bariatric surgery candidates. Specifically, we examined correlations between MMPI-2-RF scales and having a regular exercise program and then compared exercisers versus non-exercisers using relative risk ratio analyses of elevations above certain cutoffs on these scales. The results indicate that certain facets of internalizing psychopathology and somatic/cognitive complaints as measured by the MMPI-2-RF are associated with increased risk of non-exercising behavior preoperatively, as hypothesized. Of note, the associations in the RRR analyses were stronger than in the correlational analyses.

As hypothesized, elevations on EID, as well as several scales in the EID domain, were associated with lack of regular exercise, which aligns with prior research indicating that internalizing psychopathology is associated with greater perceived barriers to PA (Schuch et al., 2017; Vancampfort et al., 2016). For example, elevations on RCd led to nearly a $20 \%$ increase in risk of lack of pre-surgical exercise, as hypothesized. High scorers on RCd are often individuals who have poor self-esteem, feel discouraged, and are experiencing overall emotional discomfort (Ben-Porath \& Tellegen, 2011), which are factors that may act as barriers to being motivated to engage in exercise. RC2 also differentiated exercisers from non-exercisers, with clinical elevations increasing an individual's risk of lack of pre-surgical sedentary behavior by nearly $20 \%$ and high scores on this scale being negatively correlated with regular exercise. Specifically, RC2 is associated with descriptors such as "sleepy" and "sluggish", which may impede exercise behaviors (Tellegen, 1985).

Contrary to our hypotheses, RC7 did not differentiate exercisers from non-exercisers. However, the item content of RC7 is fairly broad in scope. Of note in this context, elevations on three RC7 scale facets (STW, AXY, and BRF) were significantly associated with lack of regular exercise, ranging from 16 to $19 \%$ increases in risk. High scorers on these scales are likely to worry about disappointments and time constraints, experience pervasive anxiety, and indicate fears that interfere with behavior, respectively. These findings coincide with research indicating that obese individuals often feel stigmatized for their weight, which may result in high self-consciousness and viewing PA in a public place as intimidating or uncomfortable (McIntosh et al., 2016; Wiklund, et al., 2011).

The HLP and NFC scales, facets of RCd, were also predictive of preoperative lack of exercise. High scorers on HLP are likely to hold the belief that they are unable to solve their problems or accomplish their goals, which is complimented by high scores on NFC and associated beliefs that one is indecisive and inefficacious. These findings align with past research, indicating that feelings of helplessness/hopelessness due to previous failed weight loss attempts, low levels of control over weight loss outcomes, and lack of selfconfidence interfere with exercise behavior (King \& Bond, 2013; Knapen et al., 2015; Piana et al., 2013; Thomas et al., 2008; Zabatiero et al., 2016). Indeed, a clinical elevation at or above $65 \mathrm{~T}$ on HLP was associated with a $37 \%$ increased risk of lack of preoperative exercise, which is the highest individual scale RRR observed in this study. Additionally, lack of self-efficacy consistently emerges as a correlate of sedentary behavior (Bandura, 2004; Trost, Owen, Bauman, Sallis, \& Brown, 2002; Williams \& French, 2011), which supports our finding of a $19 \%$ increased risk of lack of PA when NFC is clinically elevated. Contrary to our hypotheses, SFD did not differentiate exercisers from non-exercisers.

Other scales in the emotional dysfunction domain that were unexpectedly predictive of increased risk of lack of regular exercise include SUI, SAV, and INTR-r. Individuals who score high on SUI are likely to report thoughts of suicidal ideation or a recent suicide attempt, which are highly comorbid with mood and anxiety disorders (Bronisch \& Wittchen, 1994). Therefore, high levels of emotional distress, reflected in SUI elevations, may act as a barrier to exercise (Bonnet et al., 2005), which is supported by a $19 \%$ increase in sedentary behavior when clinical elevations on SUI occur.

As previously mentioned, obese individuals experience high levels of stigma, resulting in high self-consciousness and deterring these individuals from exercising in a public place (McIntosh et al., 2016; Wilund, Olsen, \& Willen, 2011). These findings may explain why elevations on INTR-r and SAV, scales associated with avoidance of social events, were associated with lack of exercise. Specifically, these associations may be driven by feelings of shame about one's weight and previous negative experiences with exercise, which have also been associated with avoidance of exercising in public (Groven \& Engelsrud, 2010; King \& Bond, 2013; Piana et al., 2013; Wiklund et al., 2011).

Regarding somatic complaints, scores on MLS were meaningfully, negatively correlated with regular exercise as hypothesized. Again, RRRs could not be included for MLS 
independently because $77 \%$ of the sample scored $55 \mathrm{~T}$ or higher on this scale. In support of these findings, bariatric surgery candidates have been found to report above-average scores on MLS in comparison with normative groups (Marek et al., 2013). Relatedly, in exploratory analyses, elevations on COG differentiated exercisers from non-exercisers. Elevations on $\mathrm{COG}$ are associated with lack of energy and difficulties concentrating. Given that obesity is associated with a reduction in cognitive functioning, elevations on this scale may be associated with perceived cognitive barriers to exercise (Wang et al., 2016). In sum, these findings align with research, indicating that bariatric candidates often experience high levels of fatigue, as well as bodily pain, chronic illness, and excessive strain on the body when exercising due to excess weight (Marek et al., 2013; Peacock et al., 2014; Zabatiero et al., 2016). Further, bariatric surgery candidates often perceive that their weight and restricted movement capabilities, in general, act as a barrier to exercising (Marek et al., 2013; Zabatiero et al., 2016).

Interestingly, elevations on both THD and RC3 were associated with greater risk of lack of regular preoperative exercise. Elevations on THD and lack of exercise may be explained by associations between thought dysfunction and unhealthy lifestyle behaviors in general (Brown, Birtwistle, Roe, \& Thompson, 1999). Finally, elevations on RC3 have been implicated in several physical and mental health problems, including chronic back pain, cardiovascular disease, and depression and anxiety (Tarescavage, Scheman, \& BenPorath, 2014).

Post hoc RRR analyses demonstrated the utility of RC2 and MLS scale combinations in predicting lack of regular exercise. These results indicate that individuals who endorse low energy and poor health, in combination with lack of positive emotions and anhedonia, are at the greatest risk of lack of exercise. These findings are particularly important because elevations on MLS alone could not be utilized in our original analyses owing to the high rate of individuals elevating this scale (Marek et al., 2013). The combinations of elevations on these scales yielded the highest estimates of increased risk of lack of regular exercise (range 37-45\%).

In terms of clinical implications, the results of our study indicate that the MMPI-2-RF can be used with improved effectiveness by clinical psychologists during pre-surgical evaluations of bariatric surgery candidates by providing useful information regarding the specific underlying causes associated with low levels of pre-surgical PA. Evaluations of MMPI-2-RF protocols by clinical psychologists in the preoperative phase may better help identify those who would benefit from interventions designed to target the underlying causes of their sedentary behavior, with aims of tailoring interventions to these causes to increase levels of PA prior to surgical interventions. For example, patients who are sedentary due to internalizing psychopathology may benefit from different exercise interventions than those who are sedentary because of somatic complaints. Exploring which interventions are best suited to an individual based on their underlying reasons for lack of exercise would be a fruitful avenue for future research. Lastly, identifying patients who are inactive and understanding why they are inactive is crucial, given that individuals who increase levels of exercise prior to surgery experience less surgical complications, greater weight loss outcomes, and more successful long-term weight maintenance than those who are physically inactive (Bond et al., 2010; Egberts, Brown, \& Brennan, 2012; Freire, Borges, Alvarez-Leite, \& Correia, 2012; King \& Bond, 2013; King et al., 2008; McCullough et al., 2006). Incorporating exercise interventions as a standard practice of care during pre-surgical evaluations is a timely topic, given that up to $41 \%$ of bariatric surgery patients have been found to be non-adherent to postsurgical PA recommendations (Elkins et al., 2005).

One notable limitation of our study was our exercise measure. First, information on regular exercise was collected via self-report during the clinical interview and therefore was susceptible to "impression management" and/or inaccurate reporting (Ambwani et al., 2013). In addition, the PA question was broad and did not account for frequency, duration, or type of exercise. Future research may implement more comprehensive self-report measures of exercise habits, as well as objective measures (e.g., accelerometers, pedometers) that can expand on the information collected in the current study. Along these same lines, it would be beneficial to explore different types of exercise as they relate to enhanced postsurgical outcomes. Finally, our measure of PA may have been susceptible to mood congruent memory bias, such that individuals reporting high scores on $\mathrm{RCd}$, for example, may tend to focus on negative aspects of behavior as a result of their negative mood state. Although our sample size was large, all bariatric candidates were from the same setting. Additional research in multiple bariatric sites throughout the country would strengthen the generalizability of our findings.

\section{Conclusions}

Overall, these results indicate that several scales in the Emotional/Internalizing Dysfunction and Somatic/Cognitive Dysfunction domains of the MMPI-2-RF may indicate whether patients are at greater risk of preoperative lack of PA. These test results can be used by psychologists conducting bariatric pre-surgical evaluations to guide treatment recommendations and better assist candidates with pre-surgical PA behavioral modifications, which are a crucial component to optimal surgery outcomes and long-term weight loss maintenance (Bond et al., 2010; Egberts et al., 
2012; King et al., 2008; King \& Bond, 2013). These results also contribute to a larger body of research that supports the use of the MMPI-2-RF in the assessment of medical, nutritional, and psychological risk factors among bariatric surgery candidates.

\section{Compliance with Ethical Standards}

Conflict of interest Yossef Ben-Porath is a paid consultant to the MMPI-2-RF publisher, the University of Minnesota Press, and distributor, Pearson. He receives royalties on sales of MMPI-2-RF materials and research grants from the MMPI-2-RF publisher. Anthony Tarescavage and Ryan Marek also receive research funding from the MMPI-2-RF publisher. Brooke Fusco and Leslie Heinberg declare that they have no conflict of interest.

Human and Animal Rights All procedures performed in studies involving human participants were in accordance with the ethical standards of the institutional and/or national research committee and with the 1964 Helsinki Declaration and its later amendments or comparable ethical standards. This article does not contain any studies with animals performed by any of the authors.

Informed Consent Informed consent was obtained from all individual participants included in the study.

\section{References}

Ambwani, S., Boeka, A. G., Brown, J. D., Byrne, T. K., Budak, A. R., Sarwer, D. B., ... O'Neil, P. M. (2013). Socially desirable responding by bariatric surgery candidates during psychological assessment. Surgery for Obesity and Related Diseases, 9, 300-305.

Anastasi, A., \& Urbina, S. (1997). Psychological testing (7th edition). Upper Saddle River: Prentice Hall/Pearson Education.

Andrade, C. (2015). Understanding relative risk, odds ratio, and related terms: As simple as it can get. The Journal of Clinical Psychiatry, $76,857-861$

Arterburn, D., Bogart, A., Coleman, K. J., Haneuse, S., Selby, J. V., Sherwood, N. E., ... O'Connor, P. J. (2013). Comparative effectiveness of bariatric surgery vs. nonsurgical treatment of type 2 diabetes among severely obese adults. Obesity Research \& Clinical Practice, 7, e258-e268.

Bandura, A. (2004). Health promotion by social cognitive means. Health Education \& Behavior, 31, 143-164.

Ben-Porath, Y. S., \& Tellegen, A. (2011). The Minnesota Multiphasic Personality Inventory-2 Restructured Form (MMPI-2-RF): Manual for administration, scoring, and interpretation. Minneapolis: University of Minnesota Press.

Bewick, V., Cheek, L., \& Ball, J. (2004). Statistics review 11: Assessing risk. Critical Care, 8, 287-291.

Bond, D. S., Jakicic, J. M., Vithiananthan, S., Thomas, J. G., Leahey, T. M., Sax, H. C., ... Wing, R. R. (2010). Objective quantification of physical activity in bariatric candidates and normal-weight controls. Surgery for Obesity and Related Diseases, 6, 72-78.

Bonnet, F., Irving, K., Terra, J. L., Nony, P., Berthezene, F., \& Moulin, P. (2005). Anxiety and depression are associated with unhealthy lifestyle in patients at risk of cardiovascular disease. Atherosclerosis, 178, 339-344.

Bronisch, T., \& Wittchen, H. U. (1994). Suicidal ideation and suicide attempts: Comorbidity with depression, anxiety disorders, and substance use disorder. European Archives of Psychiatry and Clinical Neuroscience, 244, 93-98.

Brown, S., Birtwistle, J., Roe, L., \& Thompson, C. (1999). The unhealthy lifestyle of people with schizophrenia. Psychological Medicine, 29, 697-701.

Chang, S., Stoll, C. R. T., \& Song, J. (2014). The effectiveness and risks of bariatric surgery. JAMA Surgery, 149, 275-287.

Colquitt, J. L., Pickett, K., Loveman, E., \& Frampton, G. K. (2014). Surgery for weight loss in adults. The Cochrane Library. https ://doi.org/10.1002/14651858.CD003641.pub4.

De Luca, M., Angrisani, L., Himpens, J., Busetto, L., Scopinaro, N., Weiner, R., ... Shikora, S. (2016). Indications for surgery for obesity and weight-related diseases: Position statements from the International Federation for the Surgery for Obesity and Metabolic Disorders (IFSO). Obesity Surgery, 26, 1659-1696.

Egberts, K., Brown, W. A., \& Brennan, L. (2012). Does exercise improve weight loss after bariatric surgery? A systematic review. Obesity Surgery, 22, 335-341.

Elkins, G., Whitfiled, P., Marcus, J., Symmonds, R., Rodriguez, J., \& Cook, T. (2005). Noncompliance with behavioral recommendations following bariatric surgery. Obesity Surgery, 15, 546-551.

Freire, R. H., Borges, M. C., Alvarez-Leite, J. I., \& Correia, M. I. T. D. (2012). Food quality, physical activity, and nutritional follow-up as a determinant of weight regain after Roux-en-Y gastric bypass. Nutrition, 28, 53-58.

Gloy, V. L., Briel, M., Bhatt, D. L., Kashyap, S. R., Schauer, P. R., Mingrone, G., ... Nordmann, A. J. (2013). Bariatric surgery versus non-surgical treatment for obesity: A systematic review and meta-analysis of randomized controlled trials. BMJ, 347, f5934.

Groven, K. S., \& Engelsrud, G. (2010). Dilemmas in the process of weight reduction: Exploring how women experience training as a means of losing weight. International Journal of Qualitative Studies in Health and Wellbeing. https://doi.org/10.3402/qhw. v5i2.5125.

King, W. C., Belle, S. H., Eid, G. M., Dakin, G. F., Inabnet, W. B., Mitchell, J. E., ... Wolfe, B. M. (2008). Physical activity levels of patients undergoing bariatric surgery in the longitudinal assessment of bariatric surgery (LABS) study. Surgery for Obesity and Related Diseases, 4, 721-728.

King, W. C., \& Bond, D. S. (2013). The importance of pre and postoperative physical activity counseling in bariatric surgery. Exercise and Sport Sciences Reviews, 41, 26-35.

Knapen, J., Vancampfort, D., Morien, Y., \& Marchal, Y. (2015). Exercise therapy improves both mental and physical health in patients with major depression. Disability and Rehabilitation, 37, 1490-1495.

Marek, R. J., Ben-Porath, Y. S., Windover, Tarescavage, A. M., Merrell, J., Ashton, K., ... Heinberg, L. J. (2013). Assessing psychosocial functioning of bariatric surgery candidates with the Minnesota Multiphasic Personality Inventory-2 Restructured Form (MMPI-2-RF). Obesity Surgery, 23, 1864-1873.

Marek, R. J., Tarescavage, A. M., Ben-Porath, Y. S., Ashton, K., Rish, J. M., \& Heinberg, L. J. (2015). Using presurgical psychological testing to predict 1-year appointment adherence and weight loss in bariatric surgery patients: Predictive validity and methodological considerations. Surgery for Obesity and Related Diseases, 11, 1171-1181.

McCullough, P. A., Gallagher, M. J., de Jong, A. T., Sandberg, K. R., Trivax, J. E., Alexander, D., ... Franklin, B. A. (2006). Cardiorespiratory fitness and short-term complications after bariatric surgery. Chest, 130(2), 517-525.

McIntosh, T., Hunter, D. J., \& Royce, S. (2016). Barriers to physical activity in obese adults: A rapid evidence assessment. Journal of Research in Nursing, 21, 271-287. https://doi. org/10.1177/1744987116647762. 
Mechanick, J. I., Youdim, A., Jones, D. B., Garvey, T., Hurley, D. L., McMahon, M. M., ... Brethauer, S. (2013). Clinical practice guidelines for the perioperative nutritional, metabolic, and nonsurgical support of the bariatric surgery patient-2013 update: Cosponsored by American Association of Clinical Endocrinologists, The Obesity Society, and American Society for Metabolic $\&$ Bariatric Surgery. Obesity, 21. S1-S27.

Peacock, J. C., Sloan, S. S., \& Cripps, B. (2014). A qualitative analysis of bariatric patients' post-surgical barriers to exercise. Obesity Surgery, 24, 292-298.

Peacock, J. C., \& Zizzi, S. J. (2011). An assessment of patient behavioral requirements pre- and post-surgery at accredited weight loss surgical centers. Obesity Surgery, 21, 1950-1957.

Piana, N., Battistini, D., Urbani, L., Romani, G., Fatone, C., Pazzagli, C., ... De Feo, P. (2013). Multidisciplinary lifestyle intervention in the obese: Its impact on patients' perception of the disease, food, and physical exercise. Nutrition, Metabolism, \& Cardiovascular Diseases, 23, 337-343.

Sansone, R. A., \& Sansone, L. A. (2010). Demoralization in patients with medical illness. Psychiatry (Edgmont), 7, 42-45.

Schauer, P. R., Bhatt, D. L., Kirwan, J. P., Wolski, K., Brethauer, S. A., Navaneethan, S. D., \& Kashyap, S. R. (2014). Bariatric surgery versus intensive medical therapy for diabetes-3-year outcomes. The New England Journal of Medicine, 370, 2002-2013.

Schuch, F., Vancampfort, D., Firth, J., Rosenbaum, S., Ward, P., Reichert, T., ... Stubbs, B. (2017). Physical activity and sedentary behavior in people with major depressive disorder: A systematic review and meta-analysis. Journal of Affective Disorders, 210, 139-150.

Snyder, B., Nguyen, A., Scarbourough, T., Yu, S., \& Wilson, E. (2009). Comparison of those who succeed in losing weight after bariatric surgery and those who fail. Surgical Endoscopy, 23, 2303-2306.

Sogg, S., Lauretti, J., \& West-Smith, L. (2016). Recommendations for the presurgical psychosocial evaluation of bariatric surgery patients. Surgery for Obesity and Related Diseases, 12, 731-749.

Speck, R. M., Bond, D. S., Sarwer, D. B., \& Farrer, J. T. (2014). A systematic review of musculoskeletal pain among bariatric surgery patients: Implications for physical activity and exercise. Surgery for Obesity and Related Diseases, 10, 161-170.

Streiner, D. L. (1998). Risky business: Making sense of estimates of risk. The Canadian Journal of Psychiatry, 43, 411-415.

Szoka, B., Chudziak, D., Batog, P., Macioch, T., Niewada, M., Belarbi, S., \& Wcislo, J. (2016). Bariatric surgery versus non-surgical treatment of morbid obesity: A systematic review. Value in Health, 19, A757-A576.

Tarescavage, A. M., Scheman, J., \& Ben-Porath, Y. S. (2014). Reliability and validity of the Minnesota Multiphasic Personality Restructured Form (MMPI-2-RF) in evaluations of chronic low back pain patients. Psychological Assessment, 27, 433-446.

Tarescavage, A. M., Windover, A., Ben-Porath, Y. S., Boutacoff, L. I., Marek, R. J., Ashton, K., ... Heinberg, L. J. (2013). Use of the MMPI-2-RF suicidal/death ideation and substance abuse scales in screening bariatric surgery candidates. Psychological Assessment, 25, 1384-1389.

Tarescavage, A. M., Wygant, D. B., Boutacoff, L. I., \& Ben-Porath, Y. S. (2013). Reliability, validity, and utility of the Minnesota Multiphasic Personality Inventory-2-RF (MMPI-2-RF) in assessments of bariatric surgery candidates. Psychological Assessment, $25,1179-1194$.

Tellegen, A. (1985). Structures of mood and personality and their relevance to assessing anxiety, with an emphasis on self-report. Hillsdale: Erlbaum.

Tellegen, A., \& Ben-Porath, Y. S. (2011). The Minnesota Multiphasic Personality Inventory-2 Restructured Form (MMPI-2-RF): Technical manual. Minneapolis: University of Minnesota Press.

Thomas, S. L., Hyde, J., Karunaratne, A., Kausman, R., \& Komesaroff, P. A. (2008). 'They all work when you stick to them': A qualitative investigation of dieting, weight loss, and physical exercise in obese individuals. Nutrition Journal. https://doi. org/10.1186/1475-2891-7-34.

Trost, S. G., Owen, N., Bauman, A. E., Sallis, J. F., \& Brown, W. (2002). Correlates of adults' participation in physical activity: Review and update. Medicine \& Science in Sports \& Exercise, 34, 1996-2001.

Vancampfort, D., Correll, C. U., Galling, B., Probst, M., De Hert, M., Ward, P. B., ... Stubbs, B. (2016). Diabetes mellitus in people with schizophrenia, bipolar disorder, and major depressive disorder: A systematic review and large scale meta-analysis. World Psychiatry, 15, 166-174.

Walfish, S., Vance, D., \& Fabricatore, A. N. (2007). Psychological evaluation of bariatric surgery applicants: Procedures and reasons for delay or denial of surgery. Obesity Surgery, 17(12), $1578-1583$.

Wang, C., Chan, J. S. Y., Ren, L., \& Yan, J. H. (2016). Obesity reduces cognitive and motor functions across the lifespan. Neural Plasticity. 2016, 1-13.

Wiklund, M., Olsen, M. F., \& Willen, C. (2011). Physical activity as viewed by adults with severe obesity, awaiting gastric bypass surgery. Physiotherapy Research International, 16, 179-186.

Williams, S. L., \& French, D. P. (2011). What are the most effective intervention techniques for changing physical activity self-efficacy and physical activity behavior-And are they the same? Health Education Research, 26, 308-322.

Wouters, E. J., Larsen, J. K., Zijlstra, H., van Ramshorst, B., \& Geenen, R. (2011). Physical activity after surgery for severe obesity: The role of exercise cognitions. Obesity Surgery, 21, 1894-1899.

Zabatiero, J., Hill, K., Gucciardi, D. F., Hamdorf, J. M., Taylor, S. F., Hagger, M. S., \& Smith, A. (2016). Beliefs, barriers, and facilitators to physical activity in bariatric surgery candidates. Obesity Surgery, 26, 1097-1109.

Zunker, C., \& King, W. C. (2012). Physical activity pre- and postbariatric surgery. In J. E. Mitchell \& M. de Zwaan (Eds.), Psychosocial assessment and treatment of bariatric surgery patients (pp. 131-158). New York, NY: Taylor \& Francis Group. 